\title{
Separability of rank two quantum states on multiple quantum spaces with different dimensions
}

\author{
Shao-Ming Fei ${ }^{1,}{ }^{2}$, Xiu-Hong Gao ${ }^{1}$, Xiao-Hong Wang ${ }^{1}$, Zhi-Xi Wang ${ }^{1}$, and Ke Wu ${ }^{1}$ \\ ${ }^{1}$ Department of Mathematics, Capital Normal University, Beijing, China. \\ ${ }^{2}$ Institute of Applied Mathematics, University of Bonn, 53115 Bonn, Germany
}

\begin{abstract}
We consider the separability of rank two quantum states on multiple quantum spaces with different dimensions. The sufficient and necessary conditions for separability of these multiparty quantum states are explicitly presented. A nonseparability inequality is also given, for the case where one of the eigenvectors corresponding to nonzero eigenvalues of the density matrix is maximally entangled.
\end{abstract}

Quantum entanglement is one of the most striking features of quantum phenomena [1]. It was first recognized by Schrödinger [2] and Einstein, Podolsky and Rosen [3], where a description of the world called local realism was suggested. Bell proved that the local realism implies constraints on the predictions of spin correlations in the form of inequalities (Bell's inequalities) [4]. The feature of quantum mechanics called nonlocality is one of the most apparent manifestations of quantum entanglement. Nonlocality has been given a lot of attention in foundational considerations, in the discussion of Bell type inequalities and hidden variable models, see e.g. [5]. Nonlocal correlations in quantum systems imply a kind of entanglement among the quantum subsystems. The recent development of quantum information theory showed that quantum entanglement can have important practical applications (see e.g. [6]). It is playing very important roles in quantum information processing such as quantum computation [7], quantum teleportation $[8,9,10,11]$ (for experimental realization see [12]), dense coding [13] and quantum cryptographic schemes $[14,15,16]$.

Due to interaction with environment, in real conditions one encounters mixed states rather than pure ones. They can still possess some residual entanglement. More specially, a mixed state is considered to be entangled if it is not a mixture of product states [17]. In mixed states the quantum correlations are weakened, hence the manifestations of mixed-state entanglement can be very subtle $[17,18,19]$. To investigate the structure of mixed-state entanglement some beautiful works have been done in quantifying entanglement $[20,21,22,23,24]$ for bipartite systems and multipartite systems (see e.g. [25, 26]). However most proposed measures of entanglement for bipartite systems involve extremizations which are difficult to handle analytically. For multipartite systems, one even does not know how to define the measures. Till now there is no general criterion that allows one to distinguish whether a mixed state is separable or not.

The separability of pure states for bipartite systems is quite well understood (cf. [27]). For mixed states, some progress has been achieved in understanding the separability and entanglement problem for bipartite systems (cf. [28]), e.g., the proper definition of separable and 
entangled states formulated by Werner [17], the Peres [29] criterion that all separable states necessarily have a positive partial transpose (PPT), which is further shown to be also a sufficient condition for separability in $2 \times 2$ and $2 \times 3$ systems [30,31]. Recently some new criterion are presented in $[32,33]$, which are necessary conditions for a state to be separable and complement the well-known PPT criterion in certain aspects.

An important property of entanglement is that the degree of entanglement of two parts of a quantum system remains invariant under local unitary transformations of these parts. Therefore the invariants of local unitary transformations give rise to an effective description of entanglement, especially for the study of equivalence of quantum mixed states under local unitary transformations, see e.g., [34, 35, 36, 37, 38]. In this paper, by using the invariants of local unitary transformations, we study sufficient and necessary conditions for (full) separability of higher-dimensional quantum systems on $H_{1} \otimes H_{2} \otimes \ldots \otimes H_{M}, \operatorname{dim} H_{i}=N_{i}, \quad i=1,2, \cdots, M$, which generalizes the results in [39] and [40]. A general pure state on $H_{1} \otimes H_{2} \otimes \ldots \otimes H_{M}$ is of the form

$$
\left|\Psi_{M}\right\rangle=\sum_{k=1}^{M} \sum_{i_{k}=1}^{N_{k}} a_{i_{1} i_{2} \ldots i_{M}}\left|e_{i_{1}}\right\rangle \otimes\left|f_{i_{2}}\right\rangle \otimes \ldots \otimes\left|g_{i_{M}}\right\rangle, \quad a_{i_{1} i_{2} \ldots i_{M}} \in \mathbb{C}
$$

with $\sum a_{i_{1} i_{2} \ldots i_{M}} a_{i_{1} i_{2} \ldots i_{M}}^{*}=1$ (* denoting complex conjugation) and $\left|e_{i_{1}}\right\rangle,\left|f_{i_{2}}\right\rangle, \ldots,\left|g_{i_{M}}\right\rangle$ the corresponding orthonormal basis of complex Hilbert spaces $H_{1}, H_{2}, \ldots, H_{M} \cdot\left|\Psi_{M}\right\rangle$ is said to be (fully) separable if $a_{i_{1} i_{2} \ldots i_{M}}=a_{i_{1}} a_{i_{2}} \ldots a_{i_{M}}$ for some $a_{i_{1}}, a_{i_{2}}, \ldots, a_{i_{M}} \in \mathbb{C}$. A mixed state is described by a density matrix $\rho$,

$$
\rho=\sum_{i=1}^{n} p_{i}\left|\Psi_{i}\right\rangle\left\langle\Psi_{i}\right|
$$

where $n \in \mathbb{N}, 0<p_{i} \leq 1, \sum_{i=1}^{n} p_{i}=1,\left|\Psi_{i}\right\rangle$ s are pure states of the form (1). $\rho$ is said to be separable if there exits a decomposition (2) such that all $\left|\Psi_{i}\right\rangle_{\mathrm{s}}$ are fully separable. We first present an invariant of local unitary transformation which characterizes the separability of pure states. Then we consider the separability of density matrices with rank two. The separability condition for these kinds of mixed states in arbitrary dimensions is explicitly given. In addition, we present a nonseparability inequality valid in the case where one of the eigenvectors corresponding to nonzero eigenvalues of a density matrix is maximally entangled.

We first consider the case of three different dimensional quantum spaces. Let $\mathcal{H}_{A}, \mathcal{H}_{B}$ and $\mathcal{H}_{C}$ be $M, N$ and $T$-dimensional complex Hilbert spaces respectively. We denote $\left\{\left|e_{i}\right\rangle\right\}_{i=1}^{M}$, $\left\{\left|f_{i}\right\rangle\right\}_{i=1}^{N}$ and $\left\{\left|g_{i}\right\rangle\right\}_{i=1}^{T}$ the orthonormal basis in $\mathcal{H}_{A}, \mathcal{H}_{B}$, and $\mathcal{H}_{C}$. A general pure state on $\mathcal{H}_{A} \otimes \mathcal{H}_{B} \otimes \mathcal{H}_{C}$ is of the form

$$
|\Psi\rangle=\sum_{i=1}^{M} \sum_{j=1}^{N} \sum_{k=1}^{T} a_{i j k}\left|e_{i}\right\rangle \otimes\left|f_{j}\right\rangle \otimes\left|g_{k}\right\rangle, \quad a_{i j k} \in \mathbf{C}
$$

with the normalization $\sum_{i=1}^{M} \sum_{j=1}^{N} \sum_{k=1}^{T} a_{i j k} a_{i j k}^{*}=1$. 
Let $U_{1}, U_{2}$ and $U_{3}$ denote unitary transformations on the Hilbert space $\mathcal{H}_{A}, \mathcal{H}_{B}$ and $\mathcal{H}_{C}$, respectively, such that

$$
\begin{gathered}
U_{1}\left|e_{i}\right\rangle=\sum_{j=1}^{M} b_{i j}\left|e_{j}\right\rangle, \quad U_{2}\left|f_{i}\right\rangle=\sum_{j=1}^{N} c_{i j}\left|f_{j}\right\rangle \\
U_{3}\left|g_{i}\right\rangle=\sum_{j=1}^{T} d_{i j}\left|g_{j}\right\rangle, \quad b_{i j}, c_{i j}, d_{i j} \in \mathbf{C}
\end{gathered}
$$

and $\sum_{j=1}^{M} b_{i j} b_{k j}^{*}=\delta_{i k}, \sum_{j=1}^{N} c_{i j} c_{k j}^{*}=\delta_{i k}, \sum_{j=1}^{T} d_{i j} d_{k j}^{*}=\delta_{i k}$ (with $\delta_{i k}$ the usual Kronecker's symbol ). We call a quantity an invariant associated with the state $|\Psi\rangle$ if it is invariant under all local unitary transformations of $U_{1} \otimes U_{2} \otimes U_{3}$. In [41] we have presented a way in constructing invariants by contracting the subindices in $a_{i j k}$ for multipartite quantum systems with equal dimensions. By generalizing the results in [41], the following quantities are straightforward shown to be invariants under local unitary transformations:

$$
\begin{aligned}
I_{0} & =\sum_{i=1}^{M} \sum_{j=1}^{N} \sum_{k=1}^{T} a_{i j k} a_{i j k}^{*}, & I_{1} & =\sum_{i, p=1}^{M} \sum_{j, q=1}^{N} \sum_{k, m=1}^{T} a_{i j k} a_{i j m}^{*} a_{p q m} a_{p q k}^{*}, \\
I_{2} & =\sum_{i, p=1}^{M} \sum_{j, q=1}^{N} \sum_{k, m=1}^{T} a_{i k j} a_{i m j}^{*} a_{p m q} a_{p k q}^{*}, & I_{3} & =\sum_{i, p=1}^{M} \sum_{j, q=1}^{N} \sum_{k, m=1}^{T} a_{k i j} a_{m i j}^{*} a_{m p q} a_{k p q}^{*} .
\end{aligned}
$$

For the case of a pair of qubits, the quantity concurrence characterizes the degree of entanglement [42]. This concurrence has a simple expression in terms of invariants [41]. For high dimensional multipartite quantum systems with equal dimensions, we defined a generalized concurrence which has a similar expression in terms of invariants. This generalized concurrence is generally no longer a measure of entanglement, but it characterizes the separability and maximally entanglement of a quantum state. For high dimensional multipartite quantum systems with different dimensions, there is no Schmidt-like decompositions in general. Nevertheless we can still define the following quantity to be a generalized concurrence,

$$
\begin{aligned}
C_{M N T}^{3} & =C_{M N T}^{3}(|\Psi\rangle)=\sqrt{2\left(3 I_{0}^{2}-I_{1}-I_{2}-I_{3}\right)} \\
& \left.=\sqrt{\sum\left(\left|a_{i j k} a_{p q m}-a_{i j m} a_{p q k}\right|^{2}+\left|a_{i j k} a_{p q m}-a_{i q k} a_{p j m}\right|^{2}+\left|a_{i j k} a_{p q m}-a_{p j k} a_{i q m}\right|^{2}\right.}\right)
\end{aligned}
$$

Different from the the case in $[41], C_{M N T}^{3}(|\Psi\rangle)=1$ does not mean that the quantum state $|\Psi\rangle$ is maximally entangled. But we still have

Lemma 1. $C_{M N T}^{3}(|\Psi\rangle)=0$ if and only if $|\Psi\rangle$ is separable.

Proof. If $|\Psi\rangle$ is factorizable, i.e., $a_{i j k}=a_{i} b_{j} c_{k}$, for some $a_{i}, b_{j}, c_{k} \in \mathbb{C}$, it is obvious that $C_{M N T}^{3}=0$.

Conversely, because $|\Psi\rangle \neq 0$, there exists $p_{0}, q_{0}, m_{0}$ such that $a_{p_{0} q_{0} m_{0}} \neq 0$. If $C_{M N T}^{3}=0$, from $\left|a_{i j k} a_{p q m}-a_{i j m} a_{p q k}\right|=0$ we have $a_{i j k}=a_{i j} b_{k}$, for some $a_{i j}, b_{k} \in \mathbb{C}$. From the rest terms in (4), we further get $a_{i j k}=a_{i}^{\prime} b_{j}^{\prime} c_{k}^{\prime}$, for some $a_{i}^{\prime}, b_{j}^{\prime}, c_{k}^{\prime} \in \mathbb{C}$, i.e., $|\Psi\rangle$ is separable. 
Now let $\rho$ be a rank two state on $\mathcal{H}_{A} \otimes \mathcal{H}_{B} \otimes \mathcal{H}_{C}$, with $\left|E_{1}\right\rangle,\left|E_{2}\right\rangle$ being its two orthonormal eigenvectors corresponding to the two nonzero eigenvalues:

$$
\rho=p\left|E_{1}\right\rangle\left\langle E_{1}|+q| E_{2}\right\rangle\left\langle E_{2}\right|
$$

where $q=1-p \in(0,1)$. Generally

$$
\left|E_{s_{1}}\right\rangle=\sum_{i=1}^{M} \sum_{j=1}^{N} \sum_{k=1}^{T} a_{i j k}^{s_{1}}\left|e_{i}\right\rangle \otimes\left|f_{j}\right\rangle \otimes\left|g_{k}\right\rangle, \quad a_{i j k}^{s_{1}} \in \mathbb{C}, \quad s_{1}=1,2
$$

with normalization $\sum_{i=1}^{M} \sum_{j=1}^{N} \sum_{k=1}^{T} a_{i j k}^{s_{1}}\left(a_{i j k}^{s_{1}}\right)^{*}=1$.

Using Lemma 1, we have that $|\Psi\rangle=\sum_{i=1}^{M} \sum_{j=1}^{N} \sum_{k=1}^{T} a_{i j k}\left|e_{i}\right\rangle \otimes\left|f_{j}\right\rangle \otimes\left|g_{k}\right\rangle$ is separable if and only if $C_{M N T}^{3}=0$, i.e.,

$$
a_{i j k} a_{p q m}=a_{i j m} a_{p q k}, \quad a_{i j k} a_{p q m}=a_{i q k} a_{p j m}, \quad a_{i j k} a_{p q m}=a_{p j k} a_{i q m}, \quad \forall i, j, k, p, q, m .
$$

And a vector of the form $\left|E_{1}\right\rangle+\lambda\left|E_{2}\right\rangle, \lambda \in \mathbb{C}$, is separable if and only if $C_{M N T}^{3}\left(\left|E_{1}\right\rangle+\lambda\left|E_{2}\right\rangle\right)=$ 0 . Using (6) we have that $\lambda$ is a common root of the following equation set $E q_{s}^{I}$ :

$$
\alpha_{s}^{I} \lambda^{2}+\beta_{s}^{I} \lambda+\gamma_{s}^{I}=0, \quad s=1,2,3,
$$

where

$$
\begin{aligned}
& \alpha_{1}^{I}=a_{i j k}^{2} a_{p q m}^{2}-a_{i j m}^{2} a_{p q k}^{2}, \quad \alpha_{2}^{I}=a_{i j k}^{2} a_{p q m}^{2}-a_{i q k}^{2} a_{p j m}^{2}, \quad \alpha_{3}^{I}=a_{i j k}^{2} a_{p q m}^{2}-a_{p j k}^{2} a_{i q m}^{2}, \\
& \beta_{1}^{I}=a_{i j k}^{2} a_{p q m}^{1}+a_{i j k}^{1} a_{p q m}^{2}-a_{i j m}^{2} a_{p q k}^{1}-a_{i j m}^{1} a_{p q k}^{2}, \\
& \beta_{2}^{I}=a_{i j k}^{2} a_{p q m}^{1}+a_{i j k}^{1} a_{p q m}^{2}-a_{i q k}^{2} a_{p j m}^{1}-a_{i q k}^{1} a_{p j m}^{2}, \\
& \beta_{3}^{I}=a_{i j k}^{2} a_{p q m}^{1}+a_{i j k}^{1} a_{p q m}^{2}-a_{p j k}^{2} a_{i q m}^{1}-a_{p j k}^{1} a_{i q m}^{2}, \\
& \gamma_{1}^{I}=a_{i j k}^{1} a_{p q m}^{1}-a_{i j m}^{1} a_{p q k}^{1}, \quad \gamma_{2}^{I}=a_{i j k}^{1} a_{p q m}^{1}-a_{i q k}^{1} a_{p j m}^{1}, \quad \gamma_{3}^{I}=a_{i j k}^{1} a_{p q m}^{1}-a_{p j k}^{1} a_{i q m}^{1}, \\
& I=\{i, j, k, p, q, m\}, \forall i, p \in\{1,2, \ldots, M\}, j, q \in\{1,2, \ldots, N\} \text { and } k, m \in\{1,2, \ldots, T\} .
\end{aligned}
$$

Lemma 2. If $\left|E_{2}\right\rangle$ is not separable, then $\rho$ is separable if and only if (8) have two distinct roots.

Proof. Suppose $\rho$ has a decomposition, $\rho=\sum_{t=1}^{l} p_{t}^{\prime}\left|U_{t}\right\rangle\left\langle U_{t}\right|$, with $l$ some positive integer and $0<p_{t}^{\prime}<1, \sum p_{t}^{\prime}=1,\left|U_{t}\right\rangle$ being separable, $\forall t$. We can write them as linear combinations of the two eigenvectors $\left|E_{1}\right\rangle$ and $\left|E_{2}\right\rangle$ which span the range of $\rho:\left|U_{t}\right\rangle=c_{1}^{t}\left|E_{1}\right\rangle+c_{2}^{t}\left|E_{2}\right\rangle$ (for some $\left.c_{1}^{t}, c_{2}^{t} \in \mathbb{C}\right)$. As $\left|U_{t}\right\rangle \neq 0, c_{1}^{t}, c_{2}^{t}$ can not be all 0 . Without losing generality, let $c_{1}^{t} \neq 0$. $\left|U_{t}\right\rangle$ is then of the form $\left|E_{1}\right\rangle+\lambda_{t}\left|E_{2}\right\rangle, \lambda_{t}=c_{2}^{t} / c_{1}^{t}$. From Lemma 1 we have that $\left|U_{t}\right\rangle$ is separable if and only if parameter $\lambda_{t}$ is a common root of the corresponding equation set $E q_{s}^{I}: \alpha_{s}^{I} \lambda^{2}+\beta_{s}^{I} \lambda+\gamma_{s}^{I}=0$.

Because $\left|E_{2}\right\rangle$ is not separable, not all $\lambda_{t}^{\prime} s$ can be equal, otherwise all the $U_{t}^{\prime} s$ would be constant multiples of a fixed vector, and $\rho$ would be rank 1 . On the other hand, as $\left|E_{2}\right\rangle$ is not separable, then $C_{M N T}^{3}$ is not zero. Hence there is some $I_{0}, s_{0}$ such that $\alpha_{s_{0}}^{I_{0}} \neq 0$, i.e., the relation 
$E q_{s}^{I}$ is indeed a quadratic equation. It must have exactly two roots, and so there are two values that are the only possible choices for the $\lambda_{t}^{\prime} s$. But in order that there is not only one possible choice, the above two roots must be different. And all the relations $E q_{s}^{I}$ have these two different roots.

Let $\mu_{1}, \mu_{2}$ be two distinct roots, which are common to all of the equations $E q_{s}^{I}$. Each vector $\left|U_{t}\right\rangle$ is either of the form $\left|E_{1}^{\prime}\right\rangle=\left(\left|E_{1}\right\rangle+\mu_{1}\left|E_{2}\right\rangle\right) / \sqrt{1+\left|\mu_{1}\right|^{2}}$, or of the form $\left|E_{2}^{\prime}\right\rangle=$ $\left(\left|E_{1}\right\rangle+\mu_{2}\left|E_{2}\right\rangle\right) / \sqrt{1+\left|\mu_{2}\right|^{2}}$.

Therefore we can write $\rho$ as $\rho=p^{\prime}\left|E_{1}^{\prime}\right\rangle\left\langle E_{1}^{\prime}\left|+\left(1-p^{\prime}\right)\right| E_{2}^{\prime}\right\rangle\left\langle E_{2}^{\prime}\right|$ with $0<p^{\prime}<1$. Comparing the coefficients of $\left|E_{k}\right\rangle\left\langle E_{l}\right|, k, l=1,2$, with the ones in the expression (5), we get the following two relations:

$$
\frac{p^{\prime}}{1+\left|\mu_{1}\right|^{2}}+\frac{1-p^{\prime}}{1+\left|\mu_{2}\right|^{2}}=p, \quad \frac{\mu_{1} p^{\prime}}{1+\left|\mu_{1}\right|^{2}}+\frac{\mu_{2}\left(1-p^{\prime}\right)}{1+\left|\mu_{2}\right|^{2}}=0 .
$$

Solving the above equations for $p$ and $p^{\prime}$ we get

$$
p=\left(1-\mu_{1} \mu_{2} \frac{z^{*}}{z}\right)^{-1}, \quad p^{\prime}=\frac{\mu_{2}\left(1+\left|\mu_{1}\right|^{2}\right)}{z-\mu_{1} \mu_{2} z^{*}},
$$

where $z=\mu_{2}-\mu_{1}$.

Conversely, let $\mu_{1}, \mu_{2}$ be two distinct roots, which are common to all of the equations $E q_{s}^{I}$. From above discussion we have $\rho=p^{\prime}\left|E_{1}^{\prime}\right\rangle\left\langle E_{1}^{\prime}\left|+\left(1-p^{\prime}\right)\right| E_{2}^{\prime}\right\rangle\left\langle E_{2}^{\prime}\right|$, i.e., $\rho$ is separable.

Before getting the separability criterion for general $\rho$ in (5), we first deal with the case where the coefficients $a_{i j k}^{s_{1}}$ in (6) are all real. We will use the following result:

Lemma 3. For a quadratic equation $a x^{2}+b x+c=0$ with $a, b, c \in \mathbb{R}, a \neq 0$, and roots $\alpha$, $\beta$ with $\alpha \neq \beta, \gamma=\left(\alpha^{*}-\beta^{*}\right) /(\alpha-\beta)$ is either 1 or -1 .

Theorem 1. If all $a_{i j k}^{s_{1}}$ are real, $\rho$ is separable if and only if one of the following quantities $\left(\triangle_{1}\right.$ or $\triangle_{2}$ ) is zero:

$$
\begin{gathered}
\triangle_{1}=\sum\left|\gamma_{s}^{I}-\left(1-p^{-1}\right) \alpha_{s}^{I}\right|^{2}+\sum\left|\beta_{s}^{I} \alpha_{s^{\prime}}^{I^{\prime}}-\alpha_{s}^{I} \beta_{s^{\prime}}^{I^{\prime}}\right|^{2} \\
\triangle_{2}=\sum\left|\gamma_{s}^{I}+\left(1-p^{-1}\right) \alpha_{s}^{I}\right|^{2}+\sum\left|\beta_{s}^{I}\right|^{2}
\end{gathered}
$$

or, equivalently, one of the following two sets of relations (14) or (15) hold:

$$
\begin{gathered}
\gamma_{s}^{I}=\left(1-p^{-1}\right) \alpha_{s}^{I}, \quad \beta_{s}^{I} \alpha_{s^{\prime}}^{I^{\prime}}=\alpha_{s}^{I} \beta_{s^{\prime}}^{I^{\prime}} \\
\gamma_{s}^{I}=-\left(1-p^{-1}\right) \alpha_{s}^{I}, \quad \beta_{s}^{I}=0
\end{gathered}
$$

where $s, s^{\prime}=1,2,3$, and $I, I^{\prime}=\{i, j, k, p, q, m\}, \forall i, p \in\{1,2, \ldots, M\}, j, q \in\{1,2, \ldots, N\}$, and $k, m \in\{1,2, \ldots, T\}$.

Proof. We prove the necessity part of the theorem in two cases:

Case 1. $\left|E_{2}\right\rangle$ is not separable. 
a). We get that (8) have two distinct roots from Lemma 2. These two roots are the solutions to all the relations $E q_{s}^{I}$. Consider for any $s=1,2,3, I=\{i, j, k, p, q, m\}, \forall i, p \in\{1,2, \ldots, M\}$, $j, q \in\{1,2, \ldots, N\}$, and $k, m \in\{1,2, \ldots, T\}$.

If $\alpha_{s}^{I} \neq 0$, the corresponding relation (8) is not an identity. All the quadratic equations in the set $E q_{s}^{I}$ have the same two distinct roots. From the standard theory of quadratic equations, we have

$$
\beta_{s}^{I} \alpha_{s_{0}}^{I_{0}}=\beta_{s_{0}}^{I_{0}} \alpha_{s}^{I}, \quad \gamma_{s}^{I} \alpha_{s_{0}}^{I_{0}}=\gamma_{s_{0}}^{I_{0}} \alpha_{s}^{I}
$$

If $\alpha_{s}^{I}=0$, then the equations $E q_{s}^{I}$ become identities, i.e., $\beta_{s}^{I}$ and $\gamma_{s}^{I}$ must be 0 too, because otherwise at least one of the relations $E q_{s}^{I}$ would be a linear equation, and there would be no two distinct roots. Thus in this case (16) also hold.

$b)$. Because all $a_{i j k}^{s_{1}}$ are real number, $\mu_{1}$ and $\mu_{2}$ are roots of a quadratic equation with real coefficients. From Lemma $3, \mu_{1} \mu_{2}=1-p^{-1}$ or $-\left(1-p^{-1}\right)$. Since $\mu_{1} \mu_{2}$ is real, the solution for $p^{\prime}$ in (11) implies that $\mu_{2} /\left(\mu_{2}-\mu_{1}\right)$ is real, which is possible if and only if either the roots are both real or the roots are both purely imaginary. In the first case, let $\mu_{2}>\mu_{1}$, we have $\mu_{1} \mu_{2}=1-p^{-1}$. From (11), we get the condition that $p^{\prime} \in[0,1]$, which is equivalent to $\mu_{2}>0$, $\mu_{1}<0$. In the second case, we have $\mu_{1} \mu_{2}=-\left(1-p^{-1}\right)$. The condition for having purely imaginary roots of quadratic equations gives that $\beta_{s}^{I}=0, \forall I$ and $s$.

c). Finally, we observe that $\mu_{1} \mu_{2}$ is nothing but the ratio $\gamma_{s_{0}}^{I_{0}} / \alpha_{s_{0}}^{I_{0}}$, which is either $1-p^{-1}$ or $-\left(1-p^{-1}\right)$. Therefore we conclude that either $\gamma_{s}^{I}=\left(1-p^{-1}\right) \alpha_{s}^{I}$ or $\gamma_{s}^{I}=-\left(1-p^{-1}\right) \alpha_{s}^{I}$ for any $I$ and $s=1,2,3$. Relation (12), (13) are verified.

Case 2. $\left|E_{2}\right\rangle$ is separable.

In this case from (7), we have $\alpha_{s}^{I}=0, \forall I$ and $s$. Since not all of the $\left|U_{t}\right\rangle$ can be multiples of $\left|E_{2}\right\rangle$, we must have at least one choice of $\lambda$ such that $\left|E_{1}\right\rangle+\lambda\left|E_{2}\right\rangle$ is separable. This must be a common root to all equations $E q_{s}^{I}$ as before. All these equations are now linear ones. When all $\beta_{s}^{I}=\gamma_{s}^{I}=0$, it is easy to see that $\left|E_{1}\right\rangle$ is separable. Excluding this case, we see that there is only one possible choice of $\lambda$. Then $\rho$ can be expressed as

$$
\rho=p^{\prime \prime}\left|E_{2}\right\rangle\left\langle E_{2}\right|+\left(1-p^{\prime \prime}\right) \frac{\left|E_{1}+\lambda E_{2}\right\rangle\left\langle E_{1}+\lambda E_{2}\right|}{1+|\lambda|^{2}} .
$$

That is $p^{\prime \prime}=1$, which is a contradiction. Thus, if $\left|E_{2}\right\rangle$ is separable, $\left|E_{1}\right\rangle$ must be separable too. It is clear that in this case (12) and (13) hold.

Now we prove the sufficiency part for the theorem. If (12) or (13) holds, it is clear the equations $E q_{s}^{I}$ have common roots. If $\left|E_{2}\right\rangle$ is not separable, then not all of these equations are identities. And there are at most two common roots. If (12) holds, the product of the two roots must be $1-p^{-1}<0$, so that the two roots are real and unequal. If (13) holds, the two roots must be purely imaginary. So in these two cases, we get that $\rho$ is separable in terms of Lemma 2. If $\left|E_{2}\right\rangle$ is separable, from (12) or (13) we know $\left|E_{1}\right\rangle$ is separable too and $\rho$ is separable. 
Generalizing the results in Theorem 1, we have, for the complex $a_{i j k}^{s_{1}}$,

Theorem 2. $\rho$ is separable if and only if there is $\theta \in \mathbb{R}$ such that

$$
\gamma_{s}^{I}=e^{i \theta}\left(1-p^{-1}\right) \alpha_{s}^{I}, \quad \beta_{s}^{I} \alpha_{s^{\prime}}^{I^{\prime}}=\alpha_{s}^{I} \beta_{s^{\prime}}^{I^{\prime}}
$$

where $s, s^{\prime}=1,2,3, I, I^{\prime}=\{i, j, k, p, q, m\}, \forall i, p \in\{1,2, \ldots, M\}, j, q \in\{1,2, \ldots, N\}$ and $k, m \in\{1,2, \ldots, T\}$, and

$$
\frac{\mu_{2}\left(1+\left|\mu_{1}\right|^{2}\right)}{z-\mu_{1} \mu_{2} z^{*}} \in[0,1]
$$

where $z=e^{i \theta} z^{*}, z=\mu_{2}-\mu_{1} \neq 0, \mu_{1}$ and $\mu_{2}$ are the roots of the equation $\alpha_{s}^{I} \lambda^{2}+\beta_{s}^{I} \lambda+\gamma_{s}^{I}=0$ for some $I$ and $s$ such that $\alpha_{s}^{I} \neq 0$.

Proof. The proof of necessity is similar to the proof of the corresponding part in Theorem 1. One only needs to note that since $z / z^{*}$ is of modulus 1 , a phase factor $e^{i \theta}$ appears in this case.

Now if (17) holds, it is clear that the equations $E q_{s}^{I}$ have common roots. If $\left|E_{2}\right\rangle$ is not separable, then some of the $\alpha_{s}^{I}$ are nonzero. The corresponding equations $E q_{s}^{I}$ have exactly two different roots by condition (18). Therefore $\rho$ is separable from Lemma 2. If $\left|E_{2}\right\rangle$ is separable, by (17) we know that all $\gamma_{s}^{I}$ are 0 . Hence both $\left|E_{2}\right\rangle$ and $\left|E_{1}\right\rangle$ are separable, and so is $\rho$.

Corollary 1. Let $\left|E_{2}\right\rangle$ be the maximally entangled state given by $\left|E_{2}\right\rangle=\left(1 / \sqrt{N_{1}}\right) \sum_{i=1}^{N_{1}}\left|e_{i}\right\rangle \otimes$ $\left|f_{i}\right\rangle \otimes\left|g_{i}\right\rangle$, where $N_{1}=\min \{M, N, T\}$. For any vector $\left|E_{1}\right\rangle$ which is orthogonal to $\left|E_{2}\right\rangle, \rho=$ $p\left|E_{1}\right\rangle\left\langle E_{1}|+(1-p)| E_{2}\right\rangle\left\langle E_{2}\right|$ is not separable for $0<p<1 / 2$.

Proof. Let

$$
C_{(1)}=\sqrt{\sum_{I, s}\left|\gamma_{s}^{I}\right|^{2}} \text { and } C_{(2)}=\sqrt{\sum_{I, s}\left|\alpha_{s}^{I}\right|^{2}}
$$

be the generalized concurrences associated with the states $\left|E_{1}\right\rangle$ and $\left|E_{2}\right\rangle$, respectively, where $s=$ $1,2,3, I=\{i, j, k, p, q, m\}, \forall i, p \in\{1,2, \ldots, M\}, j, q \in\{1,2, \ldots, N\}$ and $k, m \in\{1,2, \ldots, T\}$.

Suppose $\rho$ is separable. As a pure state is separable if and only if the corresponding generalized concurrence is zero, a necessary condition for separability is that $C_{(1)}$ and $C_{(2)}$ should be inversely proportional to the contribution of the corresponding pure state to $\rho$, i.e. the eigenvalue corresponding to that eigenstate. From the separable condition $\gamma_{s}^{I}=e^{i \theta}\left(1-p^{-1}\right) \alpha_{s}^{I}$, we get $C_{(1)}=\frac{1-p}{p} C_{(2)}$. As $\left|E_{2}\right\rangle$ is maximally entangled, $C_{(2)} \neq 0$, and $C_{(1)} / C_{(2)}=\frac{1-p}{p} \leq 1$, so we have $p \geq 1 / 2$, which is a contradiction.

The above approach can be extended to the case of multiquantum systems. We consider now the separability of $\left|\Psi_{M}\right\rangle$ given by (1). We have a quadratic $I_{0}=\sum a_{i_{1} i_{2} \ldots i_{M}} a_{i_{1} i_{2} \ldots i_{M}}^{*}$ and $d=2^{M-1}-1$ biquadratic invariants:

$$
I_{T S}=\sum a_{T S} a_{T S^{\prime}}^{*} a_{T^{\prime} S^{\prime}} a_{T^{\prime} S}^{*}
$$


where $T$ and $T^{\prime}$ are all possible nontrivial subset of $I=\left\{i_{1}, i_{2}, \ldots, i_{M}\right\}, I^{\prime}=\left\{\tilde{i}_{1}, \tilde{i}_{2}, \ldots, \tilde{i}_{M}\right\}$, respectively, $\forall i_{k}, \tilde{i}_{k}=1,2, \cdots, N_{k}, k=1,2, \ldots, M$, i.e.,

$$
T \neq \emptyset, \quad T \neq I, \quad S=I \backslash T ; \quad T^{\prime} \neq \emptyset, \quad T^{\prime} \neq I^{\prime}, \quad S^{\prime}=I^{\prime} \backslash T^{\prime} .
$$

$T$ and $T^{\prime}$ are subindices of $a$, associated with the same Hilbert spaces. A generalized concurrence can be defined by

$$
C_{N_{1}, N_{2}, \ldots, N_{M}}^{M}=\sqrt{2\left(d I_{0}^{2}-I_{1}-I_{2}-\cdots-I_{d}\right)}=\sqrt{\sum_{p}\left|a_{T S} a_{T^{\prime} S^{\prime}}-a_{T S^{\prime}} a_{T^{\prime} S}\right|^{2}},
$$

where $\sum_{p}$ stands for the summation over all possible combination of the indices of $T$ and $S$. Similar to Lemma 1, one can prove:

Lemma 4. $C_{N_{1}, N_{2}, \ldots, N_{M}}^{M}=0$ if and only if $\left|\Psi_{M}\right\rangle$ is separable.

Let $\rho$ be a rank two state on $H_{1} \otimes H_{2} \otimes \cdots \otimes H_{M}$, with $\left|E_{1}\right\rangle,\left|E_{2}\right\rangle$ being its two orthonormal eigenvectors corresponding to the two nonzero eigenvalues:

$$
\rho=p\left|E_{1}\right\rangle\left\langle E_{1}|+q| E_{2}\right\rangle\left\langle E_{2}\right|
$$

where $q=1-p \in(0,1)$. Generally

$$
\left|E_{s_{1}}\right\rangle=\sum_{k=1}^{M} \sum_{i_{k}=1}^{N_{k}} a_{i_{1} i_{2} \ldots i_{M}}^{s_{1}}\left|e_{i_{1}}\right\rangle \otimes\left|f_{i_{2}}\right\rangle \otimes \ldots \otimes\left|g_{i_{M}}\right\rangle, \quad a_{i_{1} i_{2} \ldots i_{M}}^{s_{1}} \in \mathbb{C}, \quad s_{1}=1,2
$$

with normalization $\sum a_{i_{1} i_{2} \ldots i_{M}}^{s_{1}}\left(a_{i_{1} i_{2} \ldots i_{M}}^{s_{1}}\right)^{*}=1$.

Using Lemma 4, we have $\left|\Psi_{M}\right\rangle$ is separable if and only if

$$
a_{T S} a_{T^{\prime} S^{\prime}}=a_{T S^{\prime}} a_{T^{\prime} S}
$$

where $T$ (resp. $T^{\prime}$ ) are all possible nontrivial subset of $I=\left\{i_{1}, i_{2}, \ldots, i_{M}\right\}$ (resp. $I^{\prime}=$ $\left.\left\{\tilde{i}_{1}, \tilde{i}_{2}, \ldots, \tilde{i}_{M}\right\}\right)$ in the sense of (19).

With the notations:

$$
\begin{aligned}
& \alpha_{T S}^{T^{\prime} S^{\prime}}=a_{T S}^{2} a_{T^{\prime} S^{\prime}}^{2}-a_{T S^{\prime}}^{2} a_{T^{\prime} S}^{2}, \quad \gamma_{T S}^{T^{\prime} S^{\prime}}=a_{T S}^{1} a_{T^{\prime} S^{\prime}}^{1}-a_{T S^{\prime}}^{1} a_{T^{\prime} S}^{1}, \\
& \beta_{T S}^{T^{\prime} S^{\prime}}=a_{T S}^{2} a_{T^{\prime} S^{\prime}}^{1}+a_{T S}^{1} a_{T^{\prime} S^{\prime}}^{2}-a_{T S^{\prime}}^{2} a_{T^{\prime} S}^{1}-a_{T S^{\prime}}^{1} a_{T^{\prime} S}^{2},
\end{aligned}
$$

we have that a vector of the form $\left|E_{1}\right\rangle+\lambda\left|E_{2}\right\rangle, \lambda \in \mathbb{C}$, is separable if and only if $\lambda$ is a common root of the following equation set:

$$
E q_{T S}^{T^{\prime} S^{\prime}}: \alpha_{T S}^{T^{\prime} S^{\prime}} \lambda^{2}+\beta_{T S}^{T^{\prime} S^{\prime}} \lambda+\gamma_{T S}^{T^{\prime} S^{\prime}}=0
$$

Similar to the case of three quantum system, one has:

Lemma 5. $\rho$ is separable if and only if (24) have two distinct roots. 
From Lemma 4 and Lemma 5 it is straightforward to prove the following conclusion:

Theorem 3. If all $a_{i_{1} i_{2} \ldots i_{M}}^{s_{1}}$ are real, $\rho$ is separable if and only if one of the following quantities $\left(\triangle_{1}\right.$ or $\left.\triangle_{2}\right)$ is zero:

$$
\begin{gathered}
\triangle_{1}=\sum\left|\gamma_{T S}^{T^{\prime} S^{\prime}}-\left(1-p^{-1}\right) \alpha_{T S}^{T^{\prime} S^{\prime}}\right|^{2}+\sum\left|\beta_{T S}^{T^{\prime} S^{\prime}} \alpha_{T_{1} S_{1}}^{T_{1}^{\prime} S_{1}^{\prime}}-\alpha_{T S}^{T^{\prime} S^{\prime}} \beta_{T_{1} S_{1}}^{T_{1}^{\prime} S_{1}^{\prime}}\right|^{2}, \\
\triangle_{2}=\sum\left|\gamma_{T S}^{T^{\prime} S^{\prime}}+\left(1-p^{-1}\right) \alpha_{T S}^{T^{\prime} S^{\prime}}\right|^{2}+\sum\left|\beta_{T S}^{T^{\prime} S^{\prime}}\right|^{2},
\end{gathered}
$$

or, equivalently, one of the following two sets of relations (27) or (28) hold:

$$
\begin{gathered}
\gamma_{T S}^{T^{\prime} S^{\prime}}=\left(1-p^{-1}\right) \alpha_{T S}^{T^{\prime} S^{\prime}}, \quad \beta_{T S}^{T^{\prime} S^{\prime}} \alpha_{T 1}^{T_{1}^{\prime} S_{1}^{\prime}}=\alpha_{T S}^{T_{S}^{\prime} S^{\prime}} \beta_{T_{1} S_{1}}^{T_{1}^{\prime} S_{1}^{\prime}}, \\
\gamma_{T S}^{T^{\prime} S^{\prime}}=-\left(1-p^{-1}\right) \alpha_{T S}^{T^{\prime} S^{\prime}}, \quad \beta_{T S}^{T^{\prime} S^{\prime}}=0,
\end{gathered}
$$

where $T$ (resp. $T^{\prime}$ ) are all possible nontrivial subset of $I=\left\{i_{1}, i_{2}, \ldots, i_{M}\right\}$, (resp. $I^{\prime}=$ $\left.\left\{\tilde{i}_{1}, \tilde{i}_{2}, \ldots, \tilde{i}_{M}\right\}\right), S=I \backslash T, S^{\prime}=I^{\prime} \backslash T^{\prime} . T_{1}$ (resp. $T_{1}^{\prime}$ ) are all possible nontrivial subset of $J=\left\{j_{1}, j_{2}, \ldots, j_{M}\right\}\left(\operatorname{resp} . J^{\prime}=\left\{\tilde{j}_{1}, \tilde{j}_{2}, \ldots, \tilde{j}_{M}\right\}\right), S_{1}=J_{1} \backslash T_{1}, S_{1}^{\prime}=J_{1}^{\prime} \backslash T_{1}^{\prime}$.

Extend Theorem 3 to general complex coefficients $a_{i_{1} i_{2} \ldots i_{M}}^{s_{1}}$, we have

Theorem 4. $\rho$ is separable if and only if there is $\theta \in \mathbb{R}$ such that

$$
\begin{gathered}
\gamma_{T S}^{T^{\prime} S^{\prime}}=e^{i \theta}\left(1-p^{-1}\right) \alpha_{T S}^{T^{\prime} S^{\prime}}, \quad \beta_{T S}^{T^{\prime} S^{\prime}} \alpha_{T_{1} S_{1}}^{T_{1}^{\prime} S_{1}^{\prime}}=\alpha_{T S}^{T^{\prime} S^{\prime}} \beta_{T_{1} S_{1}}^{T_{1}^{\prime} S_{1}^{\prime}} \\
\frac{\mu_{2}\left(1+\left|\mu_{1}\right|^{2}\right)}{z-\mu_{1} \mu_{2} z^{*}} \in[0,1] .
\end{gathered}
$$

where $T, T^{\prime}, S, S^{\prime}, T_{1}, T_{1}^{\prime}, S_{1}, S_{1}^{\prime}$ are defined as in Theorem $3, z=e^{i \theta} z^{*}, z=\mu_{2}-\mu_{1} \neq 0, \mu_{1}$ and $\mu_{2}$ are the roots of the equation $\alpha_{T S}^{T^{\prime} S^{\prime}} \lambda^{2}+\beta_{T S}^{T^{\prime} S^{\prime}} \lambda+\gamma_{T S}^{T^{\prime} S^{\prime}}=0$ for some $T, S, T^{\prime}, S^{\prime}$ such that $\alpha_{T S}^{T^{\prime} S^{\prime}} \neq 0$.

The criterion is operational. For a given rank two density matrix on $H_{1} \otimes H_{2} \otimes \cdots \otimes H_{M}$, to find its separability one only needs to calculate the two eigenvectors $\left|E_{1}\right\rangle,\left|E_{2}\right\rangle$ corresponding to the two nonzero eigenvalues and check if formula (29) is satisfied or not. The (finite) number of equations need to be checked depends on the dimensions of $H_{i}$ and $M$. For given dimensions, it increases according to $2^{M-1}-1$.

Corollary 2. Let $\left|E_{2}\right\rangle$ be the maximally entangled vector given by $\left|E_{2}\right\rangle=(1 / \sqrt{N}) \sum_{i=1}^{N}\left|e_{i}\right\rangle \otimes$ $\left|f_{i}\right\rangle \otimes \cdots \otimes\left|g_{i}\right\rangle$, where $N=\min \left\{N_{1}, N_{2}, \ldots, N_{M}\right\}$. For any vector $\left|E_{1}\right\rangle$ which is orthogonal to $\left|E_{2}\right\rangle, \rho=p\left|E_{1}\right\rangle\left\langle E_{1}|+(1-p)| E_{2}\right\rangle\left\langle E_{2}\right|$ is not separable for $0<p<1 / 2$.

We have studied the sufficient and necessary conditions for separability of rank two mixed states in higher-dimensional multipartite quantum systems on $H_{1} \otimes H_{2} \otimes \ldots \otimes H_{M}$. The separability condition for these kind of mixed states in arbitrary dimensions is explicitly presented, together with a nonseparability inequality for the case where one of the eigenvectors corresponding to nonzero eigenvalues of a density matrix is maximally entangled. 
Acknowledgement. This work has been supported by NSF of China (No. 19975061) and the National Key Project for Basic Research of China (G1998030601). We would like to thank the referee for very useful comments.

\section{References}

[1] A. Peres, Quantum Mechanics: Concepts and Methods, Kluwer, Dordrecht (1993).

[2] E. Schrodinger: Naturwissenschaften 23, 807 (1935).

[3] A. Einstein, B. Podolsky and N. Rosen: Phys. Rev. 47, 777 (1935).

[4] J. Bell, Physics 1, 195 (1964).

[5] Quantum Theory: Reconsideration of Foundations, edited by A. Khrennikov, Växjö University Press 2002.

[6] Phys. World, March 1998; J. Gruska Quantum Computing, McGraw-Hill, London (1999).

[7] See, for example, D.P. DiVincenzo, Science 270, 255 (1995).

[8] C.H. Bennett, G. Brassard, C. Crépeau, R. Jozsa, A. Peres, and W.K. Wootters, Phys. Rev. Lett. 70, 1895 (1993).

[9] S. Albeverio and S.M. Fei, Phys. Lett. A 276, 8-11 (2000).

[10] G.M. D'Ariano, P.Lo Presti, M.F. Sacchi, Phys. Lett. A 272, 32 (2000).

[11] S. Albeverio, S.M. Fei and W.L. Yang, Commun. Theor. Phys. 38, 301-304 (2002); Phys. Rev. A 66, 012301 (2002).

[12] D. Bouwmeester, J.-W. Pan, K. Mattle, M. Elbl, H. Weinfurter and A. Zeilinger, Nature (London) 390, 575 (1997);

D. Boschi, S. Brance, F. De Martini, L. Hardy and S. Popescu, Phys. Rev. Lett. 80, 1121 (1998);

A. Furusawa, J.L. Sørensen, S. L. Braunstein, C. A. Fuchs, H. J. Kimble and E. S. Polzik: Science 282, 706 (1998);

M. A. Nielsen, E. Knill and R. Laflamme: Nature 396, 52 (1998).

[13] C.H. Bennett and S.J. Wiesner, Phys. Rev. Lett. 69, 2881 (1992).

[14] A. Ekert, Phys. Rev. Lett. 67, 661 (1991).

[15] D. Deutsch, A. Ekert, R. Jozsa, C. Macchiavello, S. Popescu and A. Sanpera, Phys. Rev. Lett. 77, 2818 (1996). 
[16] C.A. Fuchs, N. Gisin, R.B. Griffiths, C-S. Niu and A. Peres, Phys. Rev. A 56, 1163 (1997).

[17] R. F. Werner, Phys. Rev. A 40, 4277 (1989).

[18] S. Popescu, Phys. Rev. Lett. 72, 797 (1994).

[19] S. Popescu, Phys. Rev. Lett. 74, 2619 (1995).

[20] C. H. Bennett, D. P. DiVincenzo, J. Smolin and W. K. Wootters: Phys. Rev. A 54, 3824 (1996).

[21] V. Vedral, M. B. Plenio, M. A. Rippin and P. L. Knight: Phys. Rev. Lett. 78, 2275 (1997).

[22] V. Vedral and M. Plenio: Phys. Rev. A 57, 1619 (1998).

[23] G. Vidal and R. Tarrach: Phys. Rev. A 59, 141 (1999).

[24] G. Vidal: J. Mod. Opt. 47, 355 (2000).

[25] M. Murao, M. B. Plenio, S. Popescu, V. Vedral and P.L. Knight: Phys. Rev. A 57, R4075 (1998); W. Dür, J. I. Cirac and R. Tarrach: Phys. Rev. Lett. 83, 3562 (1999); N. Linden, S. Popescu and A. Sudbery: ibid. 83, 243 (1999).

[26] C. H. Bennett, D. DiVincenzo, T. Mor, P. Shor, J. Smolin and B. Terhal: Phys. Rev. Lett. 82,5385 (1999).

[27] A. Peres, "Quantum Theory: Concepts and Methods", Kluwer Academic Publishers (1995).

[28] see M. Horodecki, P. Horodecki and R. Horodecki in "Quantum Information - Basic Concepts and Experiments", Eds. G. Alber and M. Weiner, (Springer, Berlin, 2000).

M. Lewenstein, D. Bruß, J. I. Cirac, B. Kraus, M. Kuś, J. Samsonowicz, A. Sanpera and R. Tarrach, J. Mod. Phys. 47, 2481 (2000).

[29] A. Peres Phys. Rev. Lett. 77, 1413 (1996).

[30] M. Horodecki, P. Horodecki and R. Horodecki, Phys. Lett. A 223, 8 (1996).

[31] P. Horodecki, Phys. Lett. A 232, 333 (1997).

[32] K. Chen and L.A. Wu, Phys. Lett. A 306, 14 (2002).

K. Chen and L.A. Wu, The Generalized Partial Transposition Criterion for Separability of Multipartite Quantum States, quant-ph/0208058.

[33] O. Rudolph, A separability criterion for density operators, J. Phys. A: Math. Gen. 33, 39513955 (2000).

O. Rudolph, Further results on the cross norm criterion for separability, quant-ph/0202121.

O. Rudolph, Some Properties of the Computable Cross Norm Criterion for Separability, quant-ph/0212047. 
[34] E.M. Rains, IEEE Transactions on Information Theory 46, 54-59 (2000).

[35] M. Grassl, M. Rötteler and T. Beth, Phys. Rev. A 58, 1833 (1998).

[36] Y. Makhlin, Nonlocal properties of two-qubit gates and mixed states and optimization of quantum computations, quant-ph/0002045.

[37] N. Linden, S. Popescu and A. Sudbery, Phys. Rev. Lett. 83, 243 (1999).

[38] S. Albeverio, S.M. Fei, P. Parashar and W.L. Yang, Nonlocal Properties and Local Invariants for Bipartite Systems, MPI 02-73, 2002.

[39] S. Albeverio, S.M. Fei and D. Goswami, Phys. Lett. A 286, 91-96 (2001).

[40] S.M. Fei, X.H. Gao, X.H. Wang, Z.X. Wang and K. Wu, Phys. Lett. A 300, 559-566 (2002).

[41] S. Albeverio and S.M. Fei, J. Opt. B: Quantum Semiclass. Opt. 3, 223 (2001).

[42] S. Hill and W.K. Wootters, Phys. Rev. Lett. 78, 5022 (1997).

W.K. Wootters, Phys. Rev. Lett. 80, 2245 (1998). 